\title{
TMEM16A alternative splicing coordination in breast cancer
}

\author{
Ifeoma Ubby ${ }^{1,4}$, Erica Bussani ${ }^{1}$, Antonio Colonna ${ }^{2}$, Giuseppe Stacul ${ }^{2}$, Martina Locatelli ${ }^{2}$, Paolo Scudieri ${ }^{3}$, \\ Luis Galietta ${ }^{3}$ and Franco Pagani ${ }^{*}$
}

\begin{abstract}
Background: TMEM16A, also known as Anoctamin-1, is a calcium-activated chloride channel gene overexpressed in many tumors. The role of TMEM16A in cancer is not completely understood and no data are available regarding the potential tumorigenic properties of the multiple isoforms generated by alternative splicing (AS).

Methods: We evaluated TMEM16A AS pattern, isoforms distribution and Splicing Coordination (SC), in normal tissues and breast cancers, through a semi-quantitative PCR-assay that amplifies transcripts across three AS exons, 6b, 13 and 15.

Results: In breast cancer, we did not observe an association either to AS of individual exons or to specific TMEM16A isoforms, and induced expression of the most common isoforms present in tumors in the HEK293 Flp-In Tet-ON system had no effect on cellular proliferation and migration. The analysis of splicing coordination, a mechanism that regulates AS of distant exons, showed a preferential association of exon $6 \mathrm{~b}$ and 15 in several normal tissues and tumors: isoforms that predominantly include exon $6 \mathrm{~b}$ tend to exclude exon 15 and vice versa. Interestingly, we found an increase in SC in breast tumors compared to matched normal tissues.

Conclusions: As the different TMEM16A isoforms do not affect proliferation or migration and do not associate with tumors, our results suggest that the resulting channel activities are not directly involved in cell growth and motility. Conversely, the observed increase in SC in breast tumors suggests that the maintenance of the regulatory mechanism that coordinates distant alternative spliced exons in multiple genes other than TMEM16A is necessary for cancer cell viability.
\end{abstract}

Keywords: TMEM16A isoforms, Alternative splicing, Splicing coordination, Breast cancer

\section{Introduction}

TMEM16A (also known as ANO1, DOG1, ORAOV2 or TAOS2) is a member of the Anoctamin family of membrane proteins, which consists of ten components (known as TMEM16A-K or ANO1-10) that share a highly conserved structure with eight transmembrane domains and cytosolic amino- and carboxy-termini domains. In 2008, three independent groups identified TMEM16A as a bona fide $\mathrm{Ca}^{2+}$-activated $\mathrm{Cl}^{-}$channel $(\mathrm{CaCC})$ essential for a variety of physiological functions including neuronal and cardiac excitation, olfactory and sensory signal transduction, vascular tone and pain perception [1,2]. TMEM16A is

\footnotetext{
* Correspondence: pagani@icgeb.org

${ }^{1}$ Human Molecular Genetics, International Centre for Genetic Engineering and Biotechnology, Trieste, Italy

Full list of author information is available at the end of the article
}

expressed in many tissues that are known to express CaCCs: bronchiolar epithelial cells, pancreatic acinar cells, proximal kidney tubule epithelium, retina, dorsal root ganglion sensory neurons, and sub- mandibular gland [3-5]. In addition to epithelia, TMEM16A is robustly expressed in interstitial cells of Cajal, which are responsible for generating pacemaker activity in smooth muscle of the gut [6-8].

TMEM16A is a sensitive biomarker for the diagnosis of gastrointestinal stromal tumors (GISTs) and it is overexpressed in several cancers including urinary bladder cancer, esophageal cancer, prostate cancer, breast cancer, head and neck squamous cell carcinoma (HNSCC), parathyroid tumors and ovarian tumors [9-13]. However, its role in tumor cell proliferation and migration is not completely understood. In cellular models, TMEM16A has

\section{Biomed Central}


been reported to positively affect cell proliferation and/or migration [9,12-16]. Conversely, Wang et al. [17] showed a negative effect of cell proliferation by preventing cell cycle transition from the G0/G1 phase to the $S$ phase via inhibition of cyclin D1 and cyclin E expression [17]. Several mechanisms have been reported that associate changes in $\mathrm{Ca}^{2+}$-dependent channel activities with its tumorigenic potential are unknown: prevention of cell cycle transition from the G0/G1 phase to the $S$ phase via inhibition of cyclin D1 and cyclin E expression [17], activation of MAPK kinases with promotion of ERK1/2 and cyclin D1 activation [9] and activation of EGFR and CAMK signalling [13]. In general, similar to other channels such as $\mathrm{Ca}(2+)$-activated $\mathrm{K}(+)$ channels and voltagegated $\mathrm{Cl}(-)$ or $\mathrm{K}(+)$ channels, TMEM16A might affect cell motility and proliferation though dynamic changes in the cellular volume [18-21].

Alternative splicing of TMEM16A can generate multiple protein isoforms with different electrophysiological properties affecting the voltage and $\mathrm{Ca}^{2+}$-dependence of the channel [22]. The most important AS occurring on TMEM16A transcripts consist in skipping/ inclusion of three exons 6b, 13 and 15. Other minor splicing events affecting exon 1, 10, 14 and 18 have also been reported, leading to the formation of several TMEM16A transcripts $[23,24]$. We have previously reported the relative percentage of inclusion of each exon relative to the total amount of TMEM16A transcripts showing that in several normal human tissues, transcripts that predominantly include exon $6 \mathrm{~b}$, also tend to lack exon 15 in the same mature transcripts and vice-versa [22]. However, the relative proportion of each isoform that take into account the real association between the AS events on the same transcript is unknown.

In the TMEM16A gene and thus on the nascent transcripts, the three exons, 6b, 13 and 15 are located relatively far apart (exons $6 \mathrm{~b}$ and 15 are located at $50 \mathrm{~kb}$ ). Comparison of data from transcript sequencing efforts, EST/cDNA sequences and microarray profiling experiments have provided evidence for AS coordination between multiple exons within a single gene [25-28]. Indeed, distant alternative splicing events on the same gene can be correlated, through a mechanism known as intragenic Splicing Coordination (SC) $[27,29,30]$. The presence of multiple splice variants on the same transcript, as found in TMEM16A, can highly increase its potential to generate multiple transcripts. However, as AS events are generally analyzed separately through PCR amplification of short regions, this correlation is difficult to establish and have not been explored in tumors. The mechanism that regulates the coordinate selection of AS events in the same gene involves both cis-acting regulatory elements present on the nascent transcripts and Polymerase II -dependent transcriptional elongation [27].
In the present study, using a PCR-assay that amplifies transcripts across the three AS exons, we found that TMEM16A AS of exons $6 \mathrm{~b}$ and 15 are coordinated in several normal tissues and that this coordination increases in breast tumors. AS of individual exons was not altered in breast tumor and overexpression of the TMEM16A isoforms in a controlled cellular system had no effect on proliferation and migration. These results suggest that TMEM16A expression and splicing does not confer a positive general effect on cell growth and motility. The unexpected increase of SC in breast tumors suggest that cancer cells maintain the regulatory mechanism that coordinates distant alternative spliced exons on the same transcript, a process that, by acting on multiple genes, may be important in tumor cell viability.

\section{Results}

\section{Identification of TMEM16A mRNA isoforms in normal} human tissues

To study the expression of the three TMEM16A AS exons, we previously used RT-PCR analysis, with primers that amplify each exon separately [22]. In normal human tissues, we observed significant changes in AS for exons $6 \mathrm{~b}$ and 15 , whereas exon 13 was minimally skipped only in two tissues (brain and skeletal muscle). In addition, several normal adult human tissues that predominantly include exon $6 \mathrm{~b}$ tend to exclude exon 15 from the mature transcript [22]. To assess the relative abundance of the TMEM16A isoforms, we set up a denaturing capillary electrophoresis of fluorescent-5' end labelled RT-PCR (Figure 1A). In this assay, the long-range PCR amplifies TMEM16A transcripts that include the three AS events. Due to the possible combinations of the three alternatively spliced exons 6b, 13 and 15, eight mRNA isoforms of different molecular size are expected to originate from the long range PCR (Figure 1B). The assignment of each peak to a specific splice variant was based upon comparison with standard molecular weight (MW) markers and direct sequencing of the bands eluted from the agarose gels. In the majority of tissues, we detected four splice variants of $725 \mathrm{bp}, 791 \mathrm{bp}, 803 \mathrm{bp}$ and $869 \mathrm{bp}$, which correspond to $6 \mathrm{~b}-13+15-, 6 \mathrm{~b}+13+15-, 6 \mathrm{~b}-13+15+$ and $6 \mathrm{~b}+13+15+$ isoforms, respectively (isoforms 2, 4, 6 and 8 in Figure 1B and Additional file 1: Figure S1). Figure $1 \mathrm{C}$ illustrates a typical electropherogram of two tissues that contains these four isoforms. As expected and previously reported [22], we observed the appearance of additional bands of $779 \mathrm{bp}$ and $713 \mathrm{bp}$ (Figure 1D), which originate from exon 13 skipping and correspond to $6 b+13-15$ - and 6b-13-15isoforms respectively (isoforms 1 and 3), in only two tissues, namely brain and skeletal muscle. The two isoforms 4 and 5 , that correspond to $6 b+13+15-$ and $6 b-13$ - and $15+$ could not be clearly distinguished by size (791 bp vs 
A

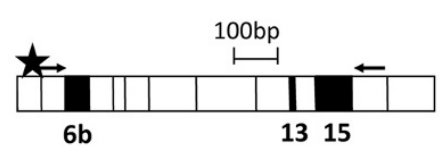

B

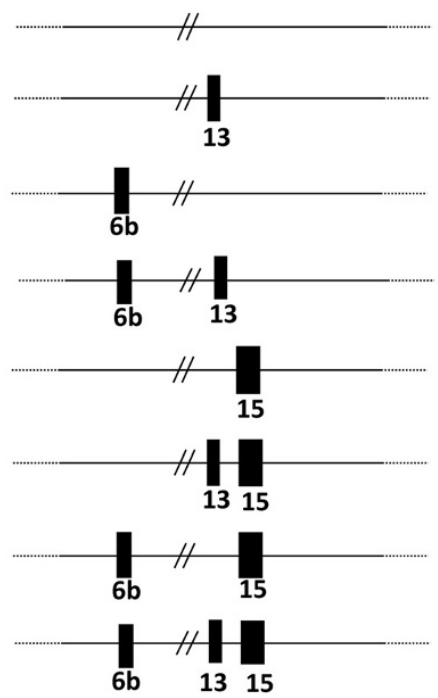

$16 b-13-15-713 b p$

$26 b-13+15-725 b p$

$36 b+13-15-779 b p$

$46 b+13+15-791 b p$

5 6b-13-15+ 792 bp

$66 b-13+15+803 b p$

$76 b+13-15+857 b p$

$86 b+13+15+869 b p$

C

Thymus

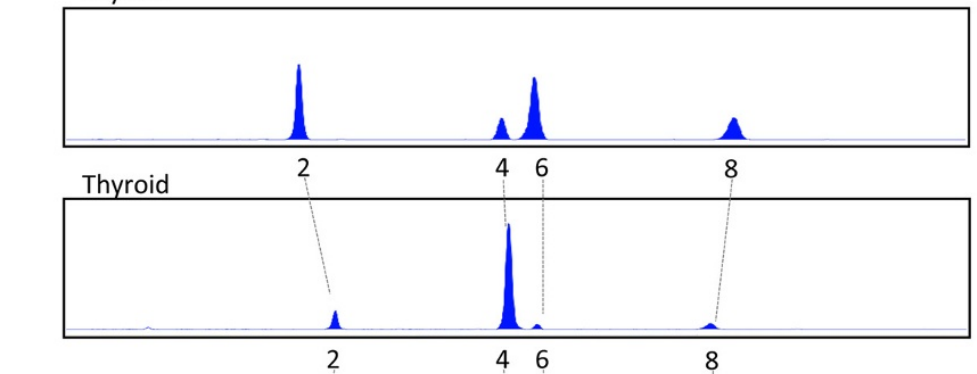

D

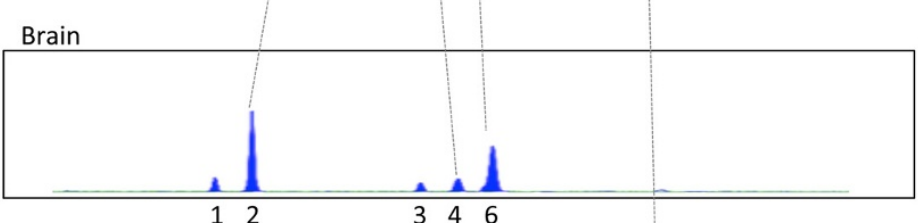

Skeletal Muscle

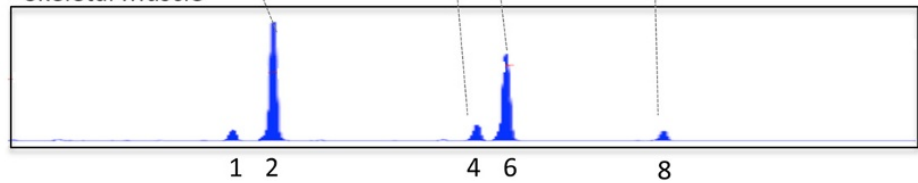

Figure 1 Identification of TMEM16A isoforms in normal adult human tissues through capillary electrophoresis. A, schematic representation of the TMEM16A mRNA showing the position of the AS exons (black boxes), and the oligonucleotide used in RT-PCR experiments. RNA was amplified with $803 \mathrm{D}^{\mathrm{FAM}}$ and $1894 \mathrm{R}$ primers. B, cartoon indicating the eight possible TMEM16A transcript variants as a result of combination of exon 6b, 13 and 15 inclusion and/or exclusion. The expected sizes of RT-PCR products are indicated on the right. C, typical electrophoregram of the RT-PCR products, evaluated by capillary electrophoresis of tissues that express isoforms with exon 13 inclusion. Each peak corresponds to the isoforms indicated in B. D, typical electrophoregram of the RT-PCR products of tissues that express the isoforms without exon13. The identity of the bands was verified by directed sequencing.

$792 \mathrm{bp})$ we assumed for quantitative purposes, that, in the samples where exon 13 is completely included, which represent the majority of tissues, they corresponds to band 4 only.
The percentage of each isoform was calculated as the area under the peaks, and Figure 2 shows the pattern of expression of the four major TMEM16A isoforms in 20 human normal adult tissues. The different peak height 


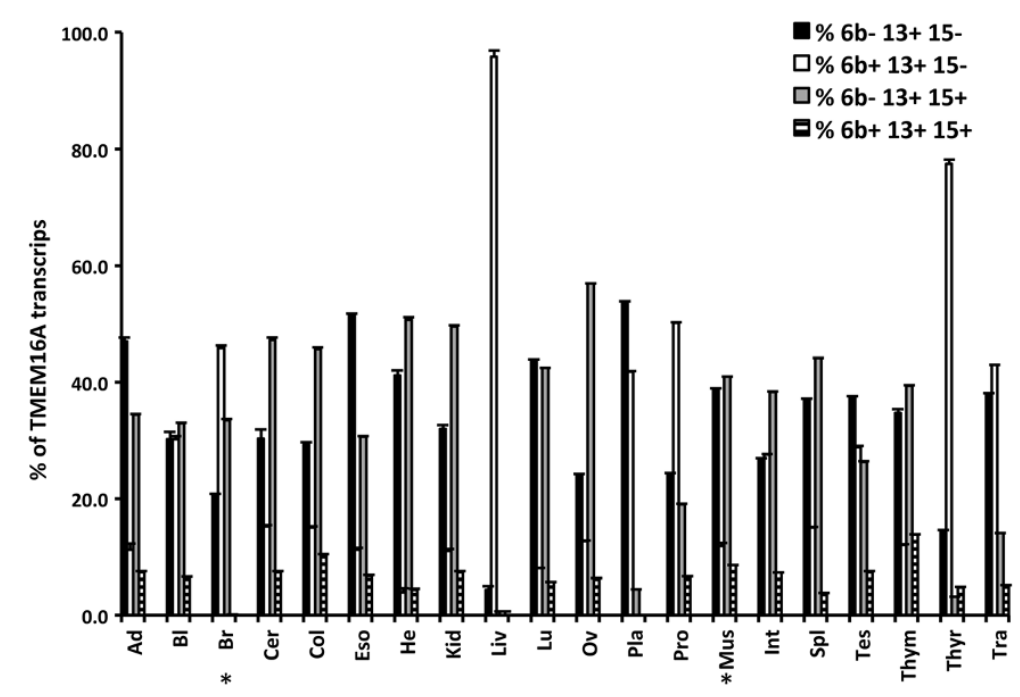

Figure 2 Quantification of TMEM16A isoforms in normal adult human tissues. TMEM16A isoforms in 20 normal adult human tissues were separated using a capillary electrophoresis system as described in Figure 1 and percentages were determined from the total area of each peak. Data are expressed as mean \pm SD of three independent experiments. ${ }^{*}$, brain and skeletal muscle showed additional minor $13-$ isoform, not reported in the graph. $\mathrm{Ad}=$ Adipose; $\mathrm{Bl}=$ Bladder; $\mathrm{Br}=$ Brain; Cer = Cervix; Col = Colon; Eso = Esophagus; He = Heart; Kid = Kidney; Liv = Liver; $\mathrm{Lu}=$ Lung; Ov = Ovary; Pla = Placenta; Pro = Prostate; Mus = Skeletal Muscle; Int = Small Intestine; Spl = Spleen; Tes = Testes; Thym = Thymus; Thyr $=$ Thyroid; Tra $=$ Trachea .

in the electrophoregram is a rough estimation of the expression levels of TMEM16A in the different tissues. The three most represented isoforms identified were $6 \mathrm{~b}-$ $13+15-, 6 b+13+15-$ and $6 b-13+15+$. Thus from this we can infer with approximation the tissue specific expression. The relative proportion of these isoforms varied significantly between tissues: for example, in lung $6 \mathrm{~b}-13+15-$ and $6 \mathrm{~b}-13+15+$ were the most expressed whereas in liver and thyroid tissues, $6 \mathrm{~b}+13+15$ - was more prevalent, representing approximately $95 \%$ and $80 \%$, of the total transcripts respectively (Figure 2 ). The isoform that contains all the three exons, $6 b+13+15+$ was present in a lower amount in all samples. To verify that in our experimental conditions, the long range PCR does not preferentially results in amplification of selected isoforms, we compared the percentage of exon inclusion calculated with the long range PCR with the percentage obtained through amplification of individual exons (Additional file 2: Figure S2A). Results shown in Additional file 2: Figure S2B, exhibited a significant correlation between the percentages of exon $6 \mathrm{~b}$ and 15 inclusions calculated with the two PCR amplification methods.

\section{TMEM16A alternative splicing of individual exons and of specific isoforms does not change in breast tumors}

To explore the role of the different TMEM16A isoforms in tumors, we next analyzed 18 breast tumors and in their corresponding normal breast tissues obtained from the same surgical specimen. The histological and receptor status of the tumors shown in Additional file 3: Table S3 and the absence of tumor cells in the controls were verified by histological analysis. Expression of TMEM16A isoforms in the 36 paired tumor-normal breast tissues was evaluated by RT-PCR amplification of transcripts across the three AS events as done for the normal human tissues (Figure 1). All the samples showed four transcripts of $725 \mathrm{bp}, 791 \mathrm{bp}, 803 \mathrm{bp}$ and $869 \mathrm{bp}$, which correspond to $6 \mathrm{~b}-13+15-, 6 \mathrm{~b}+13+15-$, $6 b-13+15+$ and $6 b+13+15+$ isoforms respectively (isoforms 2, 4, 6 and 8 in Figure 1B). No band with skipping of exon 13 was detected. The data were analyzed by considering the percentage of inclusion of each exon on the total transcript (Figure 3A) and evaluating individually the different TMEM16A isoforms (Figure 3B). In the normal breast, exon $6 \mathrm{~b}, 15$ and 13 were present in approximately $40 \%, 10 \%$ and $100 \%$ of the TMEM16A transcripts respectively and these percentages did not change significantly in the tumors (Figure 3A). Similarly, the analysis of the distribution of the four mRNA isoforms showed no significant differences between the normal samples and the tumors (Figure 3B). Most of the transcripts in both cases corresponded to $6 \mathrm{~b}-13+15$ and $6 b+13+15$ - isoforms (40-50\%). Approximately $10 \%$ of transcripts correspond to $6 \mathrm{~b}-13+15+$ isoform and $5 \%$ to the isoform that include all the exons $(6 b+13+$ $15+)$. The scatter of the standard deviation (SD) in tumor samples was increased in comparison to normal breast tissues, indicating a higher variability of the four isoforms distribution in cancer. All together these results 


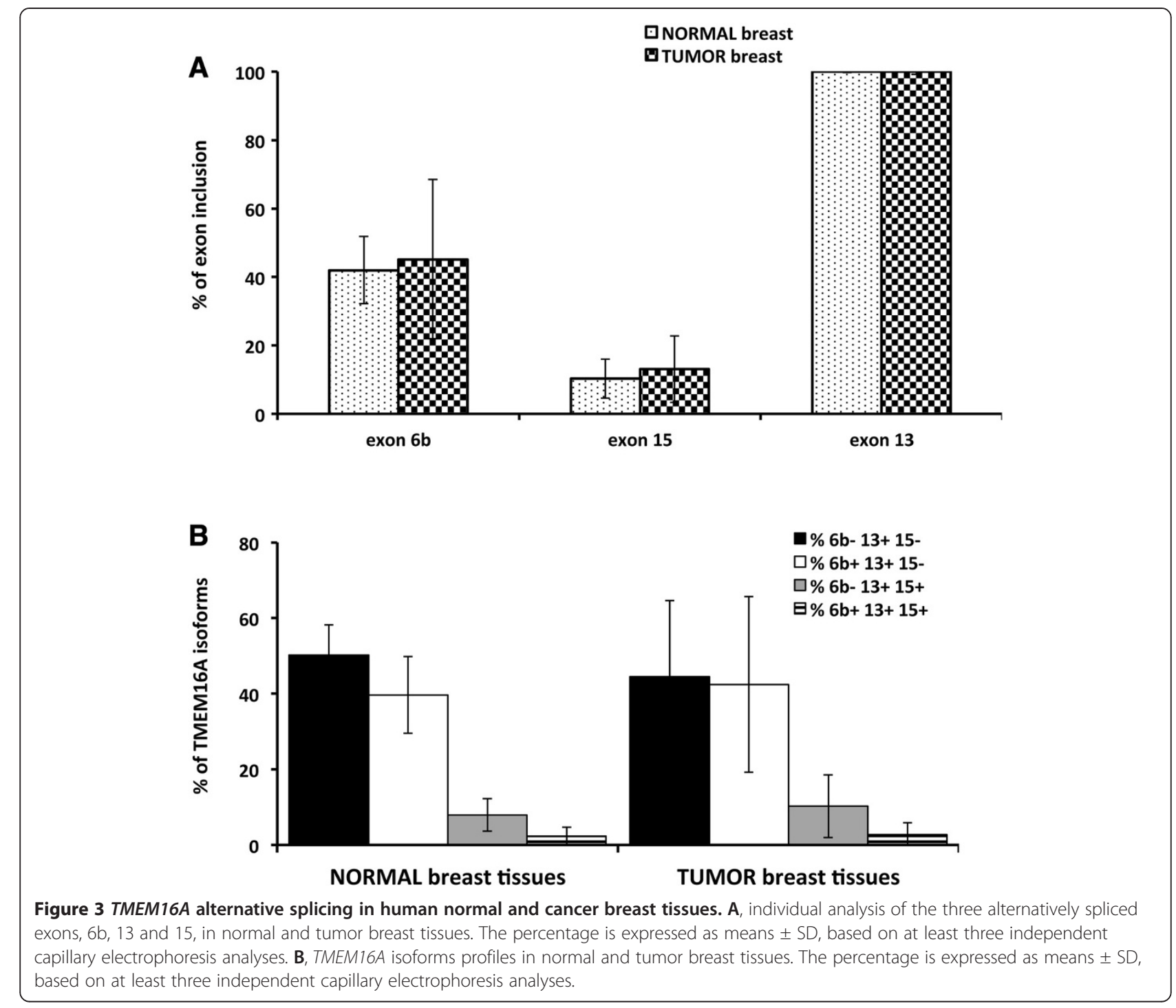

indicate that neither the individual AS events nor the derived isoforms are associated to breast tumors.

\section{Overexpression of TMEM16A isoforms in HEK293 cells do not affect cell migration and cell proliferation}

To determine the possible role of the different TMEM16A isoforms on migration and proliferation, we generated stable clones in HEK293 Flp-In cells. We selected the four most expressed isoforms detected in breast tumor samples (Figure 3B) and the additional isoform $6 b+13-15$ present in normal brain and skeletal muscle that lacks exon 13 (Figure 1). In the HEK293 Flp-In Tet-ON system, the presence of a unique copy of the transgene at the identical chromosomal site and driven by the inducible tetracycline (Tet) promoter, allow a precise and comparable evaluation of the biological properties of the different TMEM16A isoforms. In all recombinant clones, Tet addition induced expression of the corresponding TMEM isoform (Additional file 4: Figure S4). To assess TMEM16A channel activity, we used the halide-sensitive yellow fluorescent protein (HS-YFP) assay [3]. HEK293 cells transfected with the various TMEM16A isoforms responded to tetracycline treatment with a very large $\mathrm{Ca}^{2+}$-activated anion transport activity (Additional file 5: Figure S5). In contrast, control cells treated under the same conditions showed virtually no anion transport. To evaluate the effect of these TMEM16A isoforms on cell migration, we performed wound-healing assay. Confluent HEK293 cells monolayer (two for each isoform) were wounded with a sterile pipette tip (scratch assay) and wound healing (re-closure of the scratch) was followed by time lapse microscopy. Cells were incubated in the presence or absence of tetracycline for $72 \mathrm{~h}$. Clones expressing the different TMEM16A isoforms (Figure 4A) did not show any significant difference in their capacity to migrate into the wounded area compared to the 
A

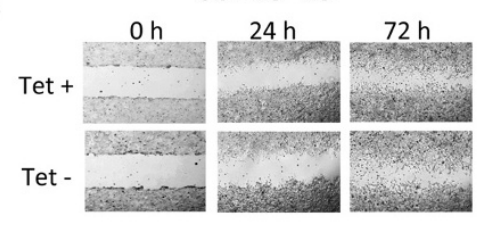

$6 b+13+15+$
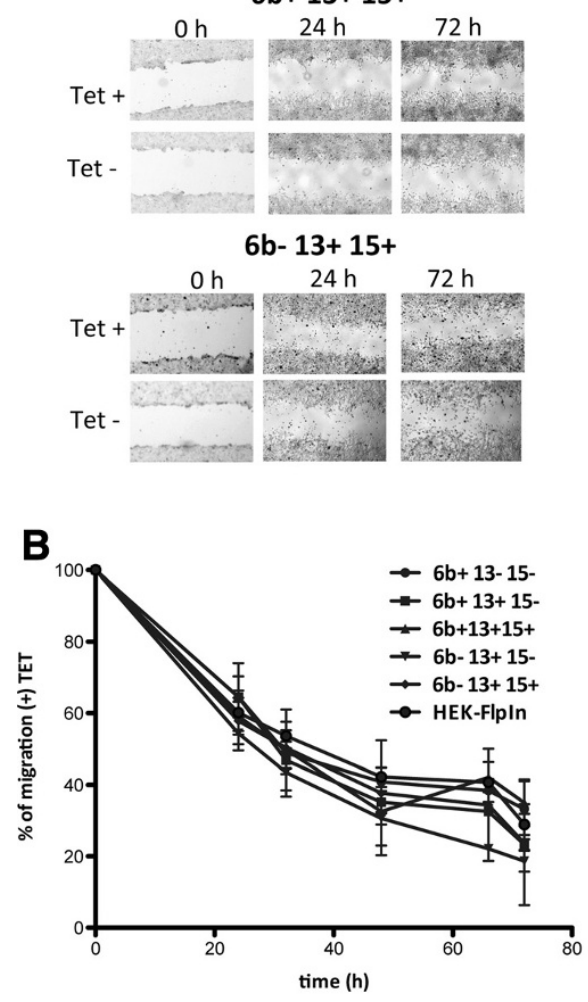

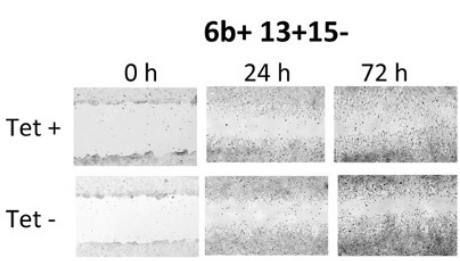

$6 b-13+15-$

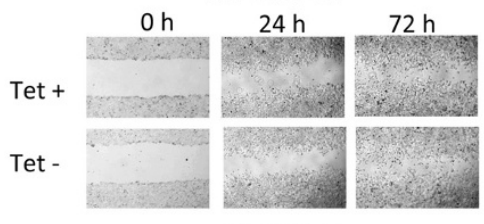

HEK-FIpIn
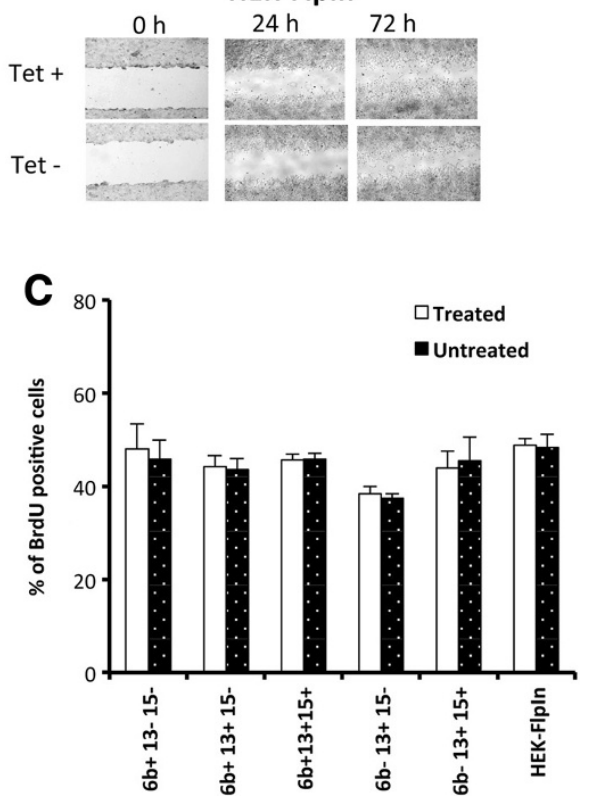

Figure 4 Cell migration and proliferation assay of TMEM16A variants. A, Representative images of wound healing in a scratch assay with inducible expression of TMEM16A variants in HEK293 cells cultured with (Tet+) or without (Tet-) tetracycline $(0.1 \mu \mathrm{g} / \mathrm{ml})$. Original magnification, 4x. (Scale bars: $5 \mu \mathrm{m})$. B, Quantification of the fraction of the wound that remains uncovered by the migratory cells as a function of time for cell treated with (Tet+) or without (Tet-). C, Cellular proliferation assay, BrdU staining of cells expressing TMEM16A variants (treated without or with tetracycline). Data represent the $\%$ of $\mathrm{BrdU}^{+}$cells and are the mean \pm SD of three independent experiments.

control HEK293 Flp-In empty cells (Figure 4A). Tet alone did not affect the percentage of migration between each clone and between the different clones (Figure 4B). We next evaluated whether overexpression of the TMEM16A isoforms could affect cell proliferation. For this purpose, we determined the incorporation of BrdU in untreated and Tet- treated HEK293 Flp-In Tet-on cells. We did not observe any significant difference in the percentage of BrdU positive cells among the different isoforms nor relative to the control cells (Figure 4C).

\section{Splicing Coordination of TMEM16A AS is higher in breast tumors}

To evaluate the relationship between the two major AS events on the same transcript and the presence of a possible intragenic Splicing Coordination (SC), we compared the percentage of exon $6 \mathrm{~b}$ inclusion in transcripts that either included exon 15 or not. Significant differences in these percentages were considered as indicative of the presence of TMEM16A isoforms in which the two AS events are coordinated. The principle of the analysis along with a graphic representation of the distribution of the TMEM16A isoforms in two representative normal tissues, cervix and adipose (a positive and a negative case of coordination, respectively) is shown in Figure 5. In these two examples, the overall percentage of exon $6 \mathrm{~b}$ inclusion is similar in cervix (23\%) and in the adipose tissue (20\%) (lane 1, in Figure 5A). However, the distribution of the exon $6 \mathrm{~b}$ abundance in $15+$ and 15- transcripts was different between them. In fact, considering 

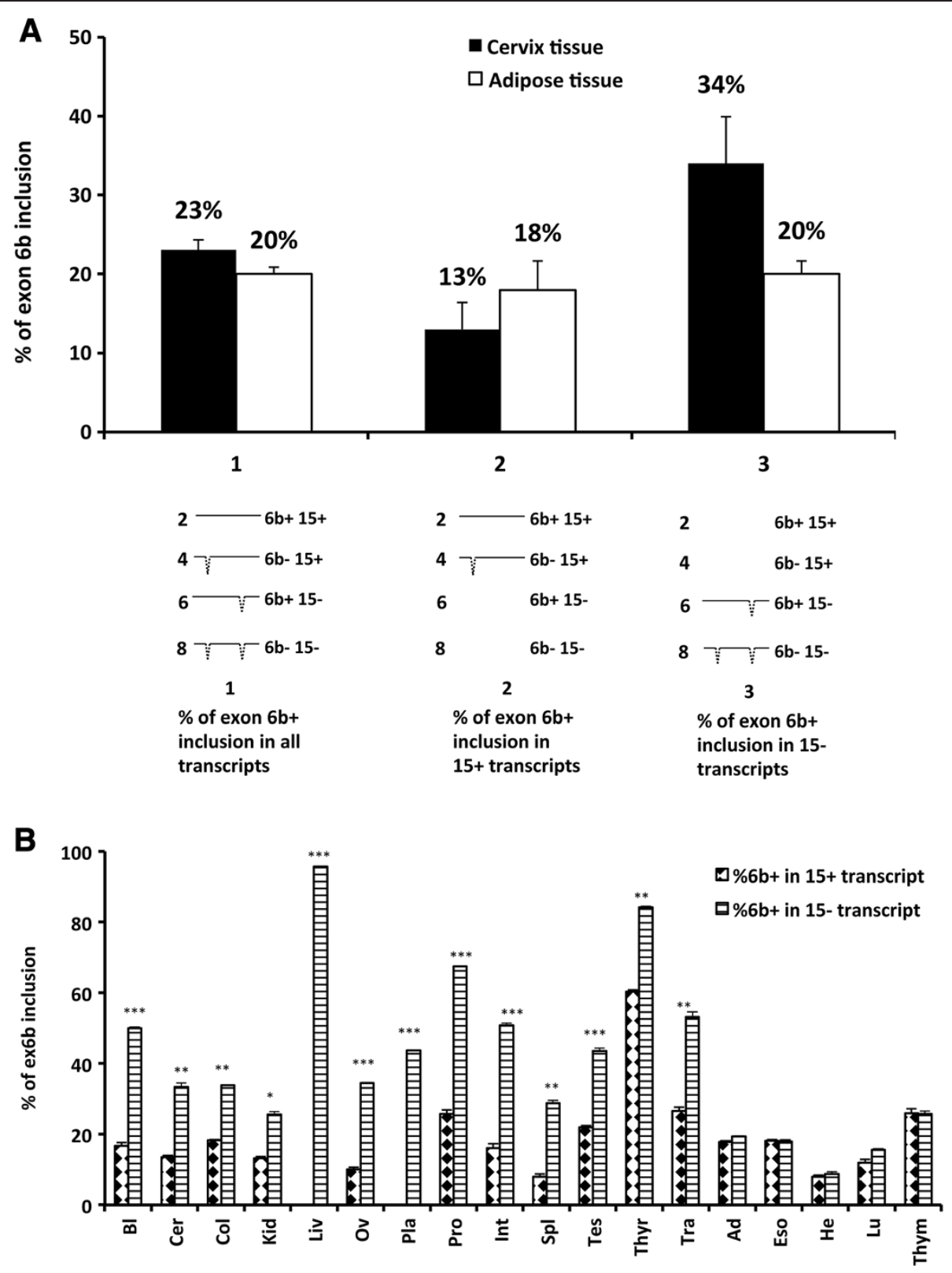

Figure 5 Splicing coordination of TMEM16A mRNA isoforms in normal adult human tissues. A, example of a positive (cervix) and a negative (adipose tissues) case of SC. The top of the panel shows the percentage of exon $6 \mathrm{~b}$ inclusion in transcripts that include or exclude exon 15. Lane 1: percentage of exon $6 \mathrm{~b}$ inclusion considering all the four isoforms. Lane 2: percentage of exon $6 \mathrm{~b}$ inclusion in the isoforms that contain only exon 15. Lane 3: percentage of exon $6 \mathrm{~b}$ inclusion in the isoforms that lacks exon 15. The identity of the isoforms considered in each calculation is depicted below each lane. B, Percentage of exon $6 \mathrm{~b}$ inclusion in transcripts that contain or lack exon 15. Quantification of exon $6 \mathrm{~b}$ inclusion associated to exon 15 inclusion or exclusion in 18 normal adult human tissues. Statistically analysis was performed using paired Student's t-test $\left({ }^{*} p<0.05 ; p<0.01^{* *}\right.$; $\mathrm{p}<0.001^{* * *}$. $\mathrm{Ad}=$ Adipose; Bl = Bladder; Cer = Cervix; Col = Colon; Eso = Esophagus; He = Heart; Kid = Kidney; Liv = Liver; Lu = Lung; Ov = Ovary; $\mathrm{Pla}=$ Placenta; Pro = Prostate; Int = Small Intestine; Spl = Spleen; Tes = Testes; Thym = Thymus; Thyr = Thyroid; Tra = Trachea.

only the isoforms that include exon 15 (lane 2), the corresponding level of inclusion of exon $6 \mathrm{~b}$ was reduced to $13 \%$ in cervix but not in adipose tissue. Similarly, in transcripts without exon 15 (lane 3), the inclusion of exon $6 \mathrm{~b}$ increased to $34 \%$ in cervix but did not change in adipose tissue. Thus, even if the total percentage of exon $6 \mathrm{~b}$ was similar in the two tissues, only one (i.e.
Cervix) showed some degree of splicing coordination. The analysis showed that exon $6 \mathrm{~b}$ has a preferential association with transcripts in which exon 15 is skipped and vice versa. The comparison of exon $6 \mathrm{~b}$ inclusion in transcripts that included exon 15 to transcript without it in 18 normal tissues showed that the two AS exons 6b and 15 were coordinated in 14 out of them. 
Coordination was evident in Bladder, Cervix, Colon, Kidney, Liver, Ovary, Placenta, Prostate, Small Intestine, Spleen, Testes, Thyroid and Trachea, although Adipose, Esophagus, Heart, Lung, and Thymus tissues were not coordinated (Figure 5B). The analysis considering the distribution of exon 15 inclusion associated to exon 6b isoforms gave comparable results (Additional file 6: Figure S6).

Next, we evaluated the TMEM16A splicing coordination in mRNA derived from normal and tumor breasts samples. This analysis revealed that in 9 out of 18 normal breast tissues (50\%), the two AS events were coordinated indicating the presence of an individual variability. Interestingly, the intragenic splicing coordination was present in 15 breast tumors (84\%) (Figure 6) and this increase in splicing coordination is due to the fact that 6 normal breasts that were not coordinated turn to be coordinated in the corresponding cancer tissues (Additional file 7: Figure S7). Thus, in TMEM16A transcripts derived from normal breast, the exon $6 \mathrm{~b}$ and 15 showed evidence of splicing coordination in $50 \%$ of the samples and this percentage increase to $84 \%$ in tumors.

\section{Discussion}

In this paper, we have evaluated the potential role in breast cancer of the different TMEM16A isoforms generated through alternative splicing. The analysis of the alternative splicing pattern in vivo in breast tumors and the functional analysis of the isoforms overexpressed in a cellular model strongly suggest that the TMEM16A $\mathrm{Ca}^{2+}$-dependent $\mathrm{Cl}^{-}$channel activities are not directly involved in tumorigenesis. More precisely, the lack of effect on HEK293 growth and motility indicates that, if TMEM16A has a role on cancer progression, it is not due to a general effect on the regulation of cell cycle and migration, as previously proposed [9,12-15]. However, this does not exclude that TMEM16A has a more cancer-specific role that is relevant to tumor growth in vivo. On the other hand, in TMEM16A transcripts derived from several normal tissues and breast tumors, inclusion/exclusion of the two distant alternatively spliced exon $6 \mathrm{~b}$ and 15 is not independent but occur in a coordinated manner on a single transcript. As this splicing coordination is significantly increased in breast tumors, the regulatory mechanism that coordinates distant alternative spliced exons on the same transcript is maintained in these cells. We speculate that maintenance of the mechanisms that control SC is necessary for regulation of AS events in other genes directly involved in tumor cell viability.

Several studies have associated TMEM16A expression with cancer [9,31-33]. However, as tumors may originate from cells that normally express TMEM16A, like GIST, which is thought to derive from the interstitial cells of Cajal [33,34], it is not clear if this channel is just an associated marker or if it directly promotes tumorigenesis. In addition, the mechanism that associates its $\mathrm{Ca}^{2+}$ dependent $\mathrm{Cl}^{-}$activity to cellular proliferation is unclear, and several studies reported contradictory effects on proliferation and/or migration. In basilar smooth muscle cells, where TMEM16A is abundantly expressed, its downregulation promotes proliferation and accordingly restoration of TMEM16A activity was suggested to be beneficial on hypertension-associated cardiovascular disease such as stroke [17]. On the contrary, other studies reported that TMEM16A promotes cell migration alone [35] or both proliferation and migration $[9,12,14,15]$. In xenografts, loss of TMEM16A through siRNA-mediated silencing was reported to inhibit tumor growth $[9,13]$. As a result of this positive effect on proliferation, and in contrast to the effect on smooth muscle cells [17], the therapeutic inhibition of TMEM16A activity was proposed for the treatment of cancers $[9,13]$. In this study, the analysis of the different TMEM16A isoforms generated through AS in vivo in breast cancer and in a controlled cellular model, does not support a direct role of the major TMEM16A isoforms in tumorigenesis. The evaluation of the different functional properties of isoforms derived from AS is not an easy task, in particular if referred to migration and proliferation. To avoid clonal variability that can affect
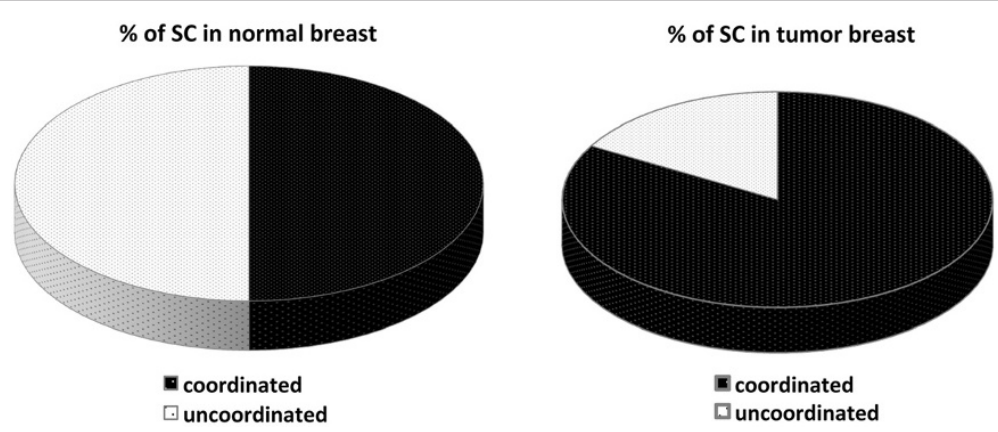

Figure 6 Alternative splicing Coordination of TMEM16A in normal and breast cancer tissues. Analysis of SC was performed in 18 normal breasts and corresponding 18 tumors. 
transgene expression due to the numbers of copies integrated and site of integration, we used the inducible HEK293 Flp-In Tet-ON system and the results clearly show that the TMEM16A isoforms per se are not sufficient to promote or inhibit proliferation and migration (Figure 4). Even if our result does not support a direct role of TMEM16A, the different isoforms might indirectly affect proliferation and migration in a cell-type specific manner. Accordingly, to affect proliferation and/or migration, TMEM16A expression would require expression of additional factors, and this association could be at the basis of the contradictory effects reported in different cell types. This cell type specificity might be critical for the development of therapeutic strategies, as different pathological conditions and target cells may differently respond to inhibition or restoration of the TMEM16A activity, as previously reported $[9,13,17]$. The HEK293 Flp-In Tet-ON system we have developed here, expressing the different isoforms in a regulated manner, can represent an interesting model to identify, with a high-throughput screening method, those factors that not only promote or inhibit proliferation in a TMEM-dependent manner but also potential AS-specific networks.

An interesting and novel aspect of our study is the identification in several normal tissues of TMEM16A SC and the observation that this coordination increases in breast tumors (Figure 6 ). In common with $\sim 25 \%$ of human genes, TMEM16A has more than one alternative splicing possibility and accordingly can generate several isoforms. Through a mechanism known as intragenic splicing coordination, $[27,28,36]$ the association between alternative spliced events is not random, as we have found for exon $6 \mathrm{~b}$ and 15 in TMEM16A. In normal breast, approximately $50 \%$ of samples showed SC, which increased to $84 \%$ in tumors. Normal mammary glands express TMEM16A [37-39] and the individual hormonal status might have an important effect on its AS pattern and $\mathrm{SC}$ thus explaining the variability observed in normal tissues. In several genes, hormones modulate AS $[40,41]$ but the effect on splicing coordination is unknown. Through histological evaluation, we do not have evidence of the presence of tumor cells in normal samples, but normal glands showed a variable relative abundance of different cell types (like epithelial, stromal and adipocytes) and this might also contribute to the individual variability.

The fact that the expression of the two AS exons $6 \mathrm{~b}$ and 15 is coordinated in the majority of TMEM16A transcripts derived from tumors (84\%), suggest that cancer progression is not associated with a relaxation of this phenomenon. The mechanisms underlying splicing coordination are largely unknown. Fibronectin (FN) [27] and slo-1 BK potassium channel [30] are well-studied examples of genes with intragenic AS coordination. In these cases, mutations that affect one AS exon had a profound effect on the other AS events, with both 5 ' to 3' polarity or bi-directionality for $F N$ and Slo-1, respectively. In addition, proper coordination of intragenic alternative splicing has been found essential for normal physiology of the slo-1 gene in vivo in Caenorhabditis elegans [30]. Based on this evidences a mechanism that lead to preferential expression of given alternate exon combination was engaged. Recruitment of splicing factors with direct interactions between the RNA-protein complexes from distinct splice sites, RNA secondary structure determinants and changes in Pol II elongation or processivity have been suggested to be involved $[27,30]$. Another interesting hypothesis to explain intragenic AS coordination might involve the formation of chromatin loops. Chromatin loops have been reported to physically link promoters to the end of the gene in order to facilitate Pol II-dependent transcription [42-45]. These loops can also occur in introns, as demonstrated for the $85-\mathrm{Kb}$ long BRCA1 gene in human cell lines and in mouse mammary tissues [31]. Interestingly, these loops change upon estrogen stimulation and during lactational development [31]. Thus it is possible that loops that put in contact distant alternatively spliced regions on the same gene might have a role in splicing coordination and possibly regulated by hormonal status. In this manner, Pol II engaged in transcription at different AS exons might physically communicate with reciprocal influence on the corresponding splicing decisions. This event might be still operative in tumor cells in order to preserve cancer viability. More deep molecular studies are required to unravel the basic mechanism of intragenic splicing coordination and understand their role in cancer.

What could be the advantage of a tumor cell to maintain an intragenic splicing coordination? As none of the TMEM16A isoforms directly affect proliferation or migration (Figure 4), it is possible that during tumorigenesis the cell would have to maintain active and efficient the mechanism involved in splicing coordination. This might not be specifically useful for the TMEM16A expression but for coordinating splicing of other genes directly involved in proliferation or apoptosis such as CD44 gene, Ron gene or FGFR2 gene [46-49].

In conclusion, this study has improved our understanding of TMEM16A splicing coordination with the identification and characterization of a non-random distribution of the mRNA isoforms in normal adult human breast tissues and tumor.

In this context, the maintenance of splicing coordination will be required for preventing a massive transcript alteration that will lead to cell death and thus the study of the basic mechanism involved might be useful for the identification of novel targets for therapeutic intervention. 


\section{Materials and methods}

\section{Human normal and primary tissue samples}

Surgically resected tissue samples were obtained under the General Surgery, "Presidio Ospedaliero di Gorizia, Italy approved by the local Ethics Committee. Primary breast tumors were obtained with the appropriate informed consent. Normal breast tissues and corresponding breast tumors were separated at the time of excision, and conserved in RNA Later (Ambion Inc) at $-20^{\circ} \mathrm{C}$. The total RNA was extracted using TRIreagent solution (Ambion) followed by an additional cleaning step (RNeasy; Qiagen). RNA was quantified using the Nanodrop spectrophotometer instrument (Thermo Scientific) and quality routinely verified on denaturing agarose gels. Normal breast tissues and tumors were classified according to standard histological evaluations, receptor and proliferation markers as reported in Additional file 3: Table S3.

\section{Analysis of TMEM16A mRNA Splicing isoforms}

One $\mu \mathrm{g}$ of total RNA derived from ductal and lobular epithelial breast tumors and normal breast samples and one $\mu \mathrm{g}$ of total RNA derived from 20 human tissues (First Choice Human Total RNA Survey Panel; Ambion) was retro-transcribed in standard conditions and amplified by PCR with a set of primers specific for each alternatively spliced exon: for exon $6 \mathrm{~b}$, the sense and antisense primers were $803 \mathrm{D}^{\mathrm{FAM}}$ and 1385R, 5' $5^{\prime}$ [6FAM]CGGAGC ACGATTGTCTATGA and 5'- GGGCCATGAAGACAG AGAAG, respectively, for exon 13, 1368D ${ }^{\text {FAM }}$ and 1525R, 5' -[6FAM]TCTCTGTCTTCATGGCCCTC and 5'-CTCC AAGACTCTGGCTTCGT, respectively, and for exon 15, $1506 \mathrm{D}^{\mathrm{FAM}}$ and 1894R, 5' -[6FAM]ACGAAGCCAGAGTC TTGGAG and 5'-GAACCGATCTCTCCATGTCAGCT TCA, respectively. Conditions for PCR were the following: $94^{\circ} \mathrm{C}$ for $5 \mathrm{~min}$ for the initial denaturation; $94^{\circ} \mathrm{C}$ for $45 \mathrm{~s}$, $58^{\circ} \mathrm{C}$ for $45 \mathrm{~s}$, and $72^{\circ} \mathrm{C}$ for $1 \mathrm{~min}$ for 35 cycles; and $72^{\circ} \mathrm{C}$ for $10 \mathrm{~min}$ for the final extension. Semi-quantitative analyses of the TMEM16A isoforms were performed by Capillary Electrophoresis $(\mathrm{CE})$ at the $\mathrm{BMR}$ genomics, Italy (http://www.bmr-genomics.it/). The relative amount of each mRNA isoform was calculated based on the corrected integrated area of each peak and size was calculated using Rox1000BV marker (Bmr-Genomics). For Spicing Coordination experiments, RNA extracted from human adult normal tissues, normal and tumor breast tissues were amplified using a labelled fluorescent $803 \mathrm{D}^{\mathrm{FAM}} 5^{\prime}$ [6FAM]CGGAGCACGATTGTCTATGA and 1894R primer 5'-GAACCGATCTCTCCATGTCAGCTTCA. Conditions for PCR were the following: $94^{\circ} \mathrm{C}$ for $3 \mathrm{~min}$ for the initial denaturation, $94^{\circ} \mathrm{C}$ for $45 \mathrm{~s}, 56^{\circ} \mathrm{C}$ for $45 \mathrm{~s}, 72^{\circ} \mathrm{C}$ for $1 \mathrm{~min}$ and $72^{\circ} \mathrm{C}$ for $10 \mathrm{~min}$ for the final extension. $1 \mu \mathrm{L}$ of the PCR product was dehydrated at the temperature of $60^{\circ} \mathrm{C}$ for $20 \mathrm{~min}$ and sent for the $\mathrm{CE}$ analysis. The results are expressed as mean \pm S.D. of three independent experiments done in duplicate.

\section{TMEM16A Expression vectors and cell culture}

The TMEM16A isoforms $6 \mathrm{~b}-13+15-, 6 \mathrm{~b}+13+15+6 \mathrm{~b}+$ $13+15-$, and $6 \mathrm{~b}+13-15$ - were obtained form corresponding plasmids $[3,22]$. TMEM16A $(6 \mathrm{~b}-13+15+)$ was generated from the TMEM16A $(6 \mathrm{~b}+13+15+$, and $6 \mathrm{~b}-13+15-)$ constructs by RT-PCR (forward) 5 '-TGCGACAAGACC TGCAGCTACTGG-3' and (reverse) 5'-TGTAGGAATT CAAACTTCAGCAG-3' and cloned in the PstI and EcoRI of pBS $(6 b-13+15-)$ to generate pBS $(6 b-13+15+)$. Subsequently, pBS $(6 b-13+15+)$ was digested with EcoRI and BamHI and cloned in the corresponding sites of pcDNA3.1 to generate pcDNA3.1 $(6 b-13+15+)$. All the coding sequences for TMEM16A $(6 \mathrm{~b}+13-15-, 6 \mathrm{~b}+13+$ $15-, \quad 6 b+13+15+, \quad 6 b-13+15-$, and $6 b-13+15+)$ were cloned in the pcDNA5 FRT/TO plasmid (Invitrogen). Stable expression of TMEM16A variants was achieved by Flp-recombinase-mediated recombination in HEK293 FlpIn cells (Invitrogen) followed by hygromycin B selection (InvivoGen). Each TMEM16A-expressing vector that expresses the Flp-recombinase (Invitrogen) was cotransfected with Effectene transfection reagent (Qiagen) and selected with a concentration of $200 \mu \mathrm{g} / \mathrm{ml}$ hygromycin B. Individual clones were obtained by limited dilution. Induction of TMEM16A isoforms expression was achieved with $0.1 \mu \mathrm{g} / \mathrm{ml}$ tetracycline (Sigma). Cells were grown in DMEM-Glutamax-I media (GIBCO) supplemented with $5 \%$ fetal bovine serum (EuroClone).

\section{In vitro wound healing assay}

Cell mobility was assessed using a scratch wound healing assay. HEK293 stable cells were plated $\left(5 \times 10^{5}\right.$ cells $)$ in $6-$ well plates, coated with poly-D-lysine $(1 \mathrm{mg} / \mathrm{ml}$, Sigma). $0.1 \mu \mathrm{g}$ tetracycline (Sigma) was added one day after plating. Confluent monolayers (24 h after tetracycline induction) were scraped with $200 \mu \mathrm{l}$ disposable plastic pipette tips. Pictures were taken every $24 \mathrm{~h}$ with a confocal microscope (Leica TCS-SL) ( $4 \times$ amplification). The distances between the wound edges were measured using GraphicConverter software. These experiments were carried out in triplicates.

\section{Immunofluorescence}

TMEM16A-inducible HEK293 cells were grown on 6well plate and cultured in the presence or absence of $0.1 \mu \mathrm{g} / \mathrm{ml}$ tetracycline for $72 \mathrm{hrs}$ to induce TMEM16A isoform expression. The cells were fixed with Bouin solution (Sigma-Aldrich) for 10 minutes at room temperature, washed twice in PBS, and incubated with blocking solution (1\% BSA and 1\% PBS) for $2 \mathrm{hrs}$ at room temperature. The cells were then stained overnight at $4^{\circ} \mathrm{C}$ with primary monoclonal antibody against 
TMEM16A (Abcam) diluted in $0.3 \%$ Triton X-100 (Sigma-Aldrich) in 1\%PBS-BSA. Cells were washed with PBS and incubated for $2 \mathrm{~h}$ with the respective secondary antibodies conjugated to Alexa Fluor-488 (Life Technologies). This was rinsed again in PBS, before viewing cells under a laser-scanning microscope LSM 5 EXCITER (Carl Zeiss MicroImaging).

\section{HS-YFP assay}

HEK-293 cells, plated in 96-well microplates, were transiently transfected with a plasmid coding for the halidsesensitive yellow fluorescent protein (HS-YFP) as previously described [3]. After 24 hours, cells were treated with tetracycline to induce TMEM16A expression. At the time of assay, cells were washed two times with $100 \mu \mathrm{l}$ PBS $(137 \mathrm{mM} \mathrm{NaCl}, 2.7 \mathrm{mM} \mathrm{KCl}, 8.1 \mathrm{mM}$ $\mathrm{Na}_{2} \mathrm{HPO}_{4}, 1.5 \mathrm{mM} \mathrm{KH} \mathrm{PO}_{4}, 1 \mathrm{mM} \mathrm{CaCl}$, and $0.5 \mathrm{mM}$ $\mathrm{MgCl}_{2} ; \mathrm{pH}=7.4$ ) and incubated for $30 \mathrm{~min}$ with $60 \mu \mathrm{l}$ PBS. Cells were transferred to the stage of a fluorescence microscope equipped with excitation and emission filters for the yellow fluorescent protein. Cell fluorescence in each well was continuously acquired with a CoolSNAP HQ2 camera before and during addition of $165 \mu \mathrm{l}$ of modified PBS $\left(\mathrm{Cl}^{-}\right.$replaced by $\mathrm{l}^{-}$; final $\mathrm{l}$ - concentration in the well: $100 \mathrm{mM}$ ) also containing $1 \mu \mathrm{M}$ ionomycin. Fluorescence cell traces were normalized to the initial, background-subtracted, fluorescence. TMEM16A activity was calculated from the maximal rate of fluorescence quenching caused by $\mathrm{l}^{-}$influx.

\section{Cell proliferation assay}

TMEM16A-inducible HEK293 cells, were grown on 96well plate and cultured in the presence or absence of $0.1 \mu \mathrm{g} / \mathrm{ml}$ tetracycline. After 24 hours, cells were incubated with EdU (Invitrogen). 30 minutes after EdU addition, cells were washed once in PBS, fixed in PFA $4 \%$, for 15 min, permeabilized with $0.5 \%$ Triton X-100 in PBS solution for $10 \mathrm{~min}$. EdU detection was carried out using Click-iT EdU Cell Proliferation Assay (Invitrogen).

\section{Statistical analysis}

A statistical analysis was performed on the investigated groups of data using the Student's t-test and the Fisher's exact test.

\section{Additional files}

Additional file 1: Figure S1. Exon-Exon junctions of TMEM16A

isoforms. Nucleotide sequences of TMEM16A cDNA isoforms expressed in human adult normal tissues, breast and tumor breast tissues.

Additional file 2: Figure S2. Correlation between the percentage of exon $6 \mathrm{~b}$ and 15 inclusion calculated in short and long RT-PCR

amplifications in normal adult human tissues. A, schematic representation of the TMEM16A mRNA showing the position of the AS exons (black boxes), and the oligonucleotide used in RT-PCR experiments. RNA was amplified with 803DFAM and 1894R; 803DFAM and 1385R; and 1506DFAM and $1894 \mathrm{R}$ primers. $B_{1}$ (Top) Correlation between the percentage of the long and the short RT-PCR amplification of TMEM16A exon 6b. (Bottom) Correlation between the percentage of the long and the short RT-PCR amplification of TMEM16A exon 15. Linear regression lines were fitted to the data points obtained from the two amplification systems. Experimental conditions were as described under Materials and Methods.

Additional file 3: Table S3. Clinicopathological variables in normal and human breast cancer samples. 36 breast samples obtained by surgical excision where divided in their normal $(N)$ and tumor $(T)$ portions. Samples were classified in virtue of their histological evaluation (second column), D defined as ductal breast or $\mathrm{L}$ defined as lobular breast samples; grading (third column), necrosis (forth column) and receptors (fifth, sixth, seventh and eighth column). ER, estrogen receptor; PgR, progesterone receptor; Ki67, nuclear proliferation marker and HER2, human epidermal growth factor receptor 2 . The histological evaluation showed 14 ductal and 3 lobular tumors and 1 ductal-bifasic. The majority of tumors are positive for the estrogen and progesterone receptor markers (15 and 13 tissues, respectively). The nuclear proliferation marker Kit67 was low $(+1$ grading) in most tumors $(n=12)$ and high (grading +2 and +3 ) in 6 tumors. The HER2 marker was detected in 5 breast cancers.

Additional file 4: Figure S4. TMEM16A isoforms expression in stably transfected HEK293 Flp- In cells. The inducible cell lines were either untreated (left panel) or tetracycline treated (right panel) prior to fluorescence measurement and examined by indirect immunofluorescence analysis using a monoclonal antiserum against TMEM16A. Original magnification, 4x. (Scale bars: 5 Mm).

Additional file 5: Figure S5. Analysis of TMEM16A function in HEK293 cells. $A$, representative cell fluorescence traces showing quenching caused by $\mathrm{Ca} 2+-$ dependent 1 - influx. The arrow shows the time of addition of the solution containing high 1 - and the $\mathrm{Ca} 2+$ ionophore ionomycin. $B$, summary of data obtained from multiple experiments. Each bar represents the anion transport (TMEM16A) activity expressed as quenching rate (QR) and calculated from the maximal slope of fluorescence decay.

Additional file 6: Figure S6. Splicing Coordination of TMEM16A mRNA isoforms in normal adult human tissues. $A$, example of a positive (cervix) and a negative (adipose tissues) case of SC. The top of the panel shows the percentage of exon $6 \mathrm{~b}$ inclusion in transcripts that include or exclude exon 15. Lane 1: percentage of exon 15 inclusion considering all the four isoforms. Lane 2: percentage of exon 15 inclusion in the isoforms that contain only exon 6b. Lane 3: percentage of exon 15 inclusion in the isoforms that lacks exon $6 \mathrm{~b}$. The identity of the isoforms considered in each calculation is depicted below each lane. B, Percentage of exon 15 inclusion in transcripts that contain or lack exon $6 \mathrm{~b}$. Quantification of exon 15 inclusion associated to exon 6b inclusion or exclusion in 20 normal adult human tissues. Statistically analysis was performed using paired Student's ttest. $\mathrm{Ad}=$ Adipose; $\mathrm{Bl}=$ Bladder; $\mathrm{Cer}=$ Cervix; $\mathrm{Col}=$ Colon; $\mathrm{EsO}=$ Esophagus; $\mathrm{He}=$ Heart; Kid = Kidney; Liv = Liver; Ov = Ovary; Pla = Placenta; Pro = Prostate; Int = Small Intestine; Spl = Spleen; Tes = Testes; Thym = Thymus; Thyr = Thyroid; Tra = Trachea.

Additional file 7: Figure S7. Alternative Splicing Coordination of TMEM16A in normal and breast cancer tissues. Quantification of exon 6b inclusion associated to exon 15 inclusion or exclusion in normal and breast cancer tissues. The former 18 samples are normal breast tissues, the latter are the corresponding breast cancer tissues. The percentage is expressed as means $\pm S D$, based on at least three independent capillary electrophoresis analyses.

Abbreviations

AS: Alternative splicing; SC: Splicing coordination.

\section{Competing interests}

The authors declare that they have no competing interests.

\section{Authors' contributions}

IU and FP conceived the idea and designed the experiments; IU, EB, PS performed the experiments; AC, GS and ML provide human breast and breast cancer tissues and performed histological analysis; IU, LG and FP wrote the manuscript. All authors read and approved the final manuscript. 


\section{Acknowledgements}

The authors thank Dr. Miguel Mano for technical assistance in cellular proliferation experiments.

This work was supported by research grants from Associazione Italiana per la Ricerca sul Cancro (AIRC) 10387 and from Fondazione Italiana Ricerca Fibrosi Cistica.

\section{Author details}

'Human Molecular Genetics, International Centre for Genetic Engineering and Biotechnology, Trieste, Italy. ${ }^{2}$ Gorizia Hospital, Presidio Ospedaliero di Gorizia, Gorizia, Italy. ${ }^{3}$ U.O.C. Genetica Medica, Istituto Giannina Gaslini, Genova, Italy. ${ }^{4}$ Present address: NCCS, National Cancer Centre Singapore, Singapore, Singapore.

Received: 8 April 2013 Accepted: 11 July 2013

Published: 16 July 2013

\section{References}

1. Brown DA, Passmore GM: Some new insights into the molecular mechanisms of pain perception. J Clin Invest 2010, 120(5):1380-1383.

2. Liu B, Linley JE, Du X, Zhang X, Ooi L, Zhang H, Gamper N: The acute nociceptive signals induced by bradykinin in rat sensory neurons are mediated by inhibition of M-type $\mathrm{K}+$ channels and activation of Ca2 + -activated Cl- channels. J Clin Invest 2010, 120(4):1240-1252

3. Caputo A, Caci E, Ferrera L, Pedemonte N, Barsanti C, Sondo E, Pfeffer U, Ravazzolo R, Zegarra-Moran O, Galietta L: TMEM16A, a membrane protein associated with calcium-dependent chloride channel activity. Science 2008, 322(5901):590-594.

4. Yang YD, Cho H, Koo JY, Tak MH, Cho Y, Shim WS, Park SP, Lee J, Lee B, Kim $B M$, et al: TMEM16A confers receptor-activated calcium-dependent chloride conductance. Nature 2008, 455(7217):1210-1215.

5. Schroeder BC, Cheng T, Jan YN, Jan LY: Expression cloning of TMEM16A as a calcium-activated chloride channel subunit. Cell 2008, 134(6):1019-1029.

6. Huang F, Rock JR, Harfe BD, Cheng T, Huang X, Jan YN, Jan LY: Studies on expression and function of the TMEM16A calcium-activated chloride channel. Proc Natl Acad Sci U S A 2009, 106(50):21413-21418.

7. Hwang SJ, Blair PJ, Britton FC, O'Driscoll KE, Hennig G, Bayguinov YR, Rock $J R$, Harfe BD, Sanders KM, Ward SM: Expression of anoctamin 1/TMEM16A by interstitial cells of Cajal is fundamental for slow wave activity in gastrointestinal muscles. J Physiol 2009, 587(Pt 20):4887-4904.

8. Zhu MH, Kim TW, Ro S, Yan W, Ward SM, Koh SD, Sanders KM: A $\mathrm{Ca}(2+)$-activated $\mathrm{Cl}(-)$ conductance in interstitial cells of Cajal linked to slow wave currents and pacemaker activity. J Physiol 2009, 587(Pt 20):4905-4918.

9. Duvvuri U, Shiwarski DJ, Xiao D, Bertrand C, Huang X, Edinger RS, Rock JR, Harfe BD, Henson BJ, Kunzelmann K, et al: TMEM16A Induces MAPK and Contributes Directly to Tumorigenesis and Cancer Progression. Cancer Res 2012, 72(13):3270-3281.

10. Carles A, Millon R, Cromer A, Ganguli G, Lemaire F, Young J, Wasylyk C, Muller D, Schultz I, Rabouel Y, et al: Head and neck squamous cell carcinoma transcriptome analysis by comprehensive validated differential display. Oncogene 2006, 25(12):1821-1831.

11. Kashyap MK, Marimuthu A, Kishore CJ, Peri S, Keerthikumar S, Prasad TS, Mahmood R, Rao S, Ranganathan P, Sanjeeviah RC, et al: Genomewide mRNA profiling of esophageal squamous cell carcinoma for identification of cancer biomarkers. Cancer Biol Ther 2009, 8(1):36-46.

12. Liu W, Lu M, Liu B, Huang Y, Wang K: Inhibition of $\mathrm{Ca}(2+)$-activated $\mathrm{Cl}(-)$ channel ANO1/TMEM16A expression suppresses tumor growth and invasiveness in human prostate carcinoma. Cancer Lett 2012, 326(1):41-51.

13. Britschgi A, Bill A, Brinkhaus H, Rothwell C, Clay I, Duss S, Rebhan M, Raman P, Guy CT, Wetzel K, et al: Calcium-activated chloride channel ANO1 promotes breast cancer progression by activating EGFR and CAMK signaling. U S A: Proc Natl Acad Sci; 2013.

14. Mazzone A, Eisenman ST, Strege PR, Yao Z, Ordog T, Gibbons SJ, Farrugia G: Inhibition of cell proliferation by a selective inhibitor of the $\mathrm{Ca}(2+)$-activated $\mathrm{Cl}(-)$ channel, Ano1. Biochem Biophys Res Commun 2012, 427(2):248-253.

15. Stanich JE, Gibbons SJ, Eisenman ST, Bardsley MR, Rock JR, Harfe BD, Ordog T. Farrugia G: Ano1 as a regulator of proliferation. Am J Physiol Gastrointest Liver Physiol 2011, 301(6):G1044-G1051.
16. Ayoub C, Wasylyk C, Li Y, Thomas E, Marisa L, Robe A, Roux M, Abecassis J, de Reynies A, Wasylyk B: ANO1 amplification and expression in HNSCC with a high propensity for future distant metastasis and its functions in HNSCC cell lines. Br J Cancer 2010, 103(5):715-726.

17. Wang M, Yang H, Zheng LY, Zhang Z, Tang YB, Wang GL, Du YH, Lv XF, Liu J, Zhou JG, et al: Downregulation of TMEM16A calcium-activated chloride channel contributes to cerebrovascular remodeling during hypertension by promoting basilar smooth muscle cell proliferation. Circulation 2012, 125(5):697-707.

18. Laniado ME, Fraser SP, Djamgoz MB: Voltage-gated K(+) channel activity in human prostate cancer cell lines of markedly different metastatic potential: distinguishing characteristics of PC-3 and LNCaP cells. Prostate 2001, 46(4):262-274.

19. Lewalle JM, Cataldo D, Bajou K, Lambert CA, Foidart JM: Endothelial cell intracellular $\mathrm{Ca} 2+$ concentration is increased upon breast tumor cell contact and mediates tumor cell transendothelial migration. Clin Exp Metastasis 1998, 16(1):21-29.

20. Soroceanu L, Manning TJ Jr, Sontheimer H: Modulation of glioma cell migration and invasion using $\mathrm{Cl}(-)$ and $\mathrm{K}(+)$ ion channel blockers. J Neurosci 1999, 19(14):5942-5954

21. Cuddapah VA, Sontheimer $\mathrm{H}$ : Ion channels and transporters [corrected] in cancer. 2. Ion channels and the control of cancer cell migration. Am J Physiol Cell Physiol 2011, 301(3):C541-C549.

22. Ferrera L, Caputo A, Ubby I, Bussani E, Zegarra-Moran O, Ravazzolo R, Pagani F, Galietta LJ: Regulation of TMEM16A chloride channel properties by alternative splicing. J Biol Chem 2009, 284(48):33360-33368.

23. O'Driscoll KE, Pipe RA, Britton FC: Increased complexity of Tmem16a/ Anoctamin 1 transcript alternative splicing. BMC Mol Biol 2011, 12:35.

24. Mazzone A, Bernard CE, Strege PR, Beyder A, Galietta L, Pasricha PJ, Rae JL, Parkman HP, Linden DR, Szurszewski JH, et al: Altered expression of Ano1 variants in human diabetic gastroparesis. J Biol Chem 2011, 286(15):13393-13403.

25. Peng T, Xue C, Bi J, Li T, Wang X, Zhang X, Li Y: Functional importance of different patterns of correlation between adjacent cassette exons in human and mouse. BMC Genomics 2008, 9:191.

26. Xing $Y$, Resch $A$, Lee $C$ : The multiassembly problem: reconstructing multiple transcript isoforms from EST fragment mixtures. Genome Res 2004, 14(3):426-441.

27. Fededa JP, Petrillo E, Gelfand MS, Neverov AD, Kadener S, Nogues G, Pelisch F, Baralle FE, Muro AF, Kornblihtt AR: A polar mechanism coordinates different regions of alternative splicing within a single gene. $\mathrm{Mo} / \mathrm{Cell}$ 2005, 19(3):393-404.

28. Fagnani M, Barash Y, Ip JY, Misquitta C, Pan Q, Saltzman AL, Shai O, Lee L, Rozenhek A, Mohammad N, et al: Functional coordination of alternative splicing in the mammalian central nervous system. Genome Biol 2007, 8(6):R108.

29. Emerick MC, Parmigiani G, Agnew WS: Multivariate analysis and visualization of splicing correlations in single-gene transcriptomes. BMC Bioinforma 2007, 8:16.

30. Glauser DA, Johnson BE, Aldrich RW, Goodman MB: Intragenic alternative splicing coordination is essential for Caenorhabditis elegans slo-1 gene function. Proc Natl Acad Sci U S A 2011, 108(51):20790-20795.

31. Simon S, Grabellus F, Ferrera L, Galietta L, Schwindenhammer B, Muhlenberg T, Taeger G, Eilers G, Treckmann J, Breitenbuecher F, et al: DOG1 Regulates Growth and IGFBP5 in Gastrointestinal Stromal Tumors. Cancer Res 2013, 73(12):3661-3670.

32. Chenevert J, Durvuri U, Chiosea S, Dacic S, Cieply K, Kim J, Shiwarski D, Seethala RR: DOG1: a novel marker of salivary acinar and intercalated duct differentiation. Mod Pathol 2012, 25(7):919-929.

33. West RB, Corless $C L$, Chen $X$, Rubin BP, Subramanian $S$, Montgomery K, Zhu $S$, Ball CA, Nielsen TO, Patel R, et al: The novel marker, DOG1, is expressed ubiquitously in gastrointestinal stromal tumors irrespective of KIT or PDGFRA mutation status. Am J Pathol 2004, 165(1):107-113.

34. Espinosa I, Lee CH, Kim MK, Rouse BT, Subramanian S, Montgomery K, Varma S, Corless CL, Heinrich MC, Smith KS, et al: A novel monoclonal antibody against DOG1 is a sensitive and specific marker for gastrointestinal stromal tumors. Am J Surg Pathol 2008, 32(2):210-218.

35. Ruiz C, Martins JR, Rudin F, Schneider S, Dietsche T, Fischer CA, Tornillo L, Terracciano LM, Schreiber R, Bubendorf $L$, et al: Enhanced expression of ANO1 in head and neck squamous cell carcinoma causes cell migration and correlates with poor prognosis. PLOS One 2012, 7(8):e43265. 
36. Johnson BE, Glauser DA, Dan-Glauser ES, Halling DB, Aldrich RW, Goodman MB: Alternatively spliced domains interact to regulate $B K$ potassium channel gating. Proc Natl Acad Sci U S A 2011, 108(51):20784-20789.

37. Ferrera L, Caputo A, Galietta L: TMEM16A protein: a new identity for $\mathrm{Ca}(2+)$-dependent $\mathrm{Cl}(-)$ channels. Physiology (Bethesda) 2010, 25(6):357-363.

38. Ousingsawat J, Martins JR, Schreiber R, Rock JR, Harfe BD, Kunzelmann K: Loss of TMEM16A causes a defect in epithelial $\mathrm{Ca} 2+-$ dependent chloride transport. J Biol Chem 2009, 284(42):28698-28703.

39. Hartzell HC, Yu K, Xiao Q, Chien LT, Qu Z: Anoctamin/TMEM16 family members are $\mathrm{Ca} 2+-$ activated $\mathrm{Cl}$ - channels. J Physiol 2009, 587(Pt 10):2127-2139.

40. Faustino NA, Cooper TA: Pre-mRNA splicing and human disease. Genes Dev 2003, 17(4):419-437.

41. Tarn WY: Cellular signals modulate alternative splicing. J Biomed Sci 2007, 14(4):517-522.

42. Sen M, Thomas SM, Kim S, Yeh Jl, Ferris RL, Johnson JT, Durvuri U, Lee J, Sahu N, Joyce S, et al: First-in-human trial of a STAT3 decoy oligonucleotide in head and neck tumors: implications for cancer therapy. Cancer Discov 2012, 2(8):694-705.

43. Subramanian S, West RB, Corless CL, Ou W, Rubin BP, Chu KM, Leung SY, Yuen ST, Zhu S, Hernandez-Boussard T, et al: Gastrointestinal stromal tumors (GISTs) with KIT and PDGFRA mutations have distinct gene expression profiles. Oncogene 2004, 23(47):7780-7790.

44. O'Sullivan JM, Tan-Wong SM, Morillon A, Lee B, Coles J, Mellor J, Proudfoot $\mathrm{NJ}$ : Gene loops juxtapose promoters and terminators in yeast. Nat Genet 2004, 36(9):1014-1018.

45. Luco RF, Pan Q, Tominaga K, Blencowe BJ, Pereira-Smith OM, Misteli T: Regulation of alternative splicing by histone modifications. Science 2010, 327(5968):996-1000.

46. Herrlich P, Morrison H, Sleeman J, Orian-Rousseau V, Konig H, Weg-Remers $\mathrm{S}$, Ponta $\mathrm{H}$ : CD44 acts both as a growth- and invasiveness-promoting molecule and as a tumor-suppressing cofactor. Ann N Y Acad Sci 2000, 910:106-118. discussion 118-120.

47. Cheng C, Sharp PA: Regulation of CD44 alternative splicing by SRm 160 and its potential role in tumor cell invasion. Mol Cell Biol 2006, 26(1):362-370

48. Ghigna C, Giordano S, Shen H, Benvenuto F, Castiglioni F, Comoglio PM, Green MR, Riva S, Biamonti G: Cell motility is controlled by SF2/ASF through alternative splicing of the Ron protooncogene. Mol Cell 2005, 20(6):881-890

49. Wagh PK, Peace BE, Waltz SE: Met-related receptor tyrosine kinase Ron in tumor growth and metastasis. Adv Cancer Res 2008, 100:1-33.

doi:10.1186/1476-4598-12-75

Cite this article as: Ubby et al.: TMEM16A alternative splicing

coordination in breast cancer. Molecular Cancer 2013 12:75.

\section{Submit your next manuscript to BioMed Central and take full advantage of:}

- Convenient online submission

- Thorough peer review

- No space constraints or color figure charges

- Immediate publication on acceptance

- Inclusion in PubMed, CAS, Scopus and Google Scholar

- Research which is freely available for redistribution 